\title{
Geriatric patients: AGGIR PATHOS SOCIOS, a tool to take into account the specificity of the geriatric patient in hospital financing complementary to APRDRG
}

\section{Julian Perelman}

\author{
Address: National School of Public Health, Nova University of Lisbon, Avenida Padre Cruz, Lisbon, 1600-560, Portugal \\ Email: Julian Perelman - jperelman@ensp.unl.pt
}

from 24th Patient Classification Systems International (PCSI) Working Conference

Lisbon, Portugal. 8-II October 2008

Published: 27 November 2008

BMC Health Services Research 2008, 8(Suppl I):A20 doi:I0.II86/I472-6963-8-SI-A20

This abstract is available from: http://www.biomedcentral.com/I472-6963/8/SI/A20

(c) 2008 Perelman; licensee BioMed Central Ltd.

\section{Introduction}

Most developed countries use case-mix systems (APRDRG-type) to define the prospective budgets of their hospitals. However, these case-mix systems are based mainly on the main diagnosis and procedures and do not take into account the specificity of geriatric patients. In the present study, we use all geriatric tools (RAI, RUGs, RIM, etc.) to evaluate the needs of acute geriatric hospitals in terms of resource use. Then, using these techniques, we design a financing model for acute geriatric hospital departments.

\section{Methods}

The AGGIR PATHOS SOCIOS (APS) tool was selected. This tool was developed by the French Society of Geriatry and Gerontology. For each patient, it collects for their dependency level according to eight criteria (eating, coherence, orientation, dressing, etc.), their pathological condition, their care profile (vital, close and episodic medical supervision, psychiatric supervision, intensive and normal rehabilitation, palliative care, etc.) and their social complexity. This tool allows a description of the profile of geriatric units in terms of patient dependency, pathological condition, care profile, social complexity and the relative workload of the unit for the different healthcare professionals (physicians, nurses, psychiatrists, physiotherapists, occupational and speech therapists, etc.). In Belgium, 77 geriatric units (1856 patients) collected the APS data by patient during two days in 2005 .

\section{Results}

The analysis of the data shows that, controlling for APRDRG and severity, the geriatric specificities have important and complementary explanatory power on length of stay and intensity of nursing, rehabilitation and physician care. Profiles of care and social complexity have the bigger impact on length of stay.

\section{Conclusion}

It is crucial to take these specificities into account in addition to APRDRGs in the hospital financing system, and define contracts with hospitals in order to avoid adverse selection and decreases in quality of care for geriatric patients. Based on these results, we propose a new financing system for acute geriatric departments. 